\title{
UWB, miniaturized and directive metamaterial loaded antenna for satellite applications
}

\author{
Parul Dawar ${ }^{1}$, N.S. Raghava ${ }^{2}$, Asok De ${ }^{3}$ \\ ${ }^{1}$ Department of Electronics and communications Engineering, Guru Tegh Bahadur Institute of Technology, \\ G-8 Area, Rajouri Garden, \\ New Delhi, India \\ E-mail: paru.dawar@gmail.com \\ ${ }^{2,3}$ Department of Electronics and Communication Engineering, Delhi Technological University, \\ Shahbad Daulatpur, Main Bawana Road \\ New Delhi, India \\ E-mail:ns.raghava@gmail.com
}

\begin{abstract}
Antenna parameter optimization using S-shaped metamaterial embedded in antenna substrate at high frequency $(\mathrm{THz})$ is elucidated in this paper. Upon incorporation of proposed metamaterials' array inside the antenna substrate, bandwidth of antenna increases by $770 \mathrm{GHz}$ and directivity by about $11 \%$. Results obtained are well in coherence between FEM based Ansoft HFSS simulation and Matlab programming based on CAD formulas using equivalent circuit analysis of patch antenna. A low frequency (scaled) prototype of the proposed structure is fabricated and nearly $15 \%$ deviation is obtained in comparison with the simulation results.
\end{abstract}

Keywords: Antenna, Metamaterial, Negative refractive index, RMPA, HFSS.

\section{Introduction}

'Metamaterials' (MTMs) are engineered to modify the bulk permeability and/or permittivity of the medium [1]. They are realized by placing periodically, structures that alter the material parameters, with elements of size less than the wavelength of the incoming electromagnetic wave. This results in "meta" i.e. "altered" behavior or behavior unattainable by natural materials [2]. Particle shape has effect on the value of negative permittivity corresponding to Frohlich resonance. The geometry of negative permittivity particle has a strong effect on its surface plasmonic properties.

The software tool HFSS is used because it is a high performance full wave electromagnetic (EM) field simulator for arbitrary 3D volumetric passive device modeling [3].

This paper abridges the design of RMPA with resonant frequency $43 \mathrm{THz}$ and operating frequency range of $38 \mathrm{THz}$ to $48 \mathrm{THz}$ having RT Duroid $\left(\varepsilon_{\mathrm{r}}=2.33\right)$ as substrate material in Section 2 by using Finite Element Method based Ansoft HFSS software and 
parametric study using equivalent circuit analysis in Matlab. Section 3 describes DNG MTM (S shaped) having negative refraction in the same frequency range. Section 4 mathematically demonstrates the resonant frequency of S- shaped MTM using internal inductances and capacitances from circuit diagram. Section 5 elucidates upon its application in antenna parameter optimization by embedding it in substrate. Section 6 concludes the trade-offs in achieving bandwidth enhancement in RMPA with DNG material in substrate.

\section{Rectangular Microstrip Patch Antenna Design}

\subsection{Design}

RMPA i.e. Rectangular Microstrip Patch Antenna, as the name implies consists of a rectangular patch over a microstrip substrate. Its major disadvantage is relatively low-impedance bandwidth which limits the field of application of these antennas. Metamaterials are embedded in the middle of its substrate to increase the bandwidth of antenna without altering its parameters and without much effecting the antenna's radiation properties. Henceforth, metamaterial based antennas are introduced.

Transmission Line model represents RMPA as two slots of width, w, and height, h, separated by transmission line of length 1 . Thus, it is a nonhomogeneous structure made up pf two dielectrics i.e. substrate and air. This shows that substrate and air will have different phase velocity and the dominant mode of propagation will be quasi-TEM. Therefore, effective permittivity, $\varepsilon_{\text {eff }}$, comes into consideration. There is fringing effect at the edges of patch, due to which the patch appears to be longer. So, Leff, i.e. effective length is defined which is obtained by adding $2 \Delta \mathrm{l}$ (additional length $\Delta \mathrm{l}$ due to fringing on each end) to the length obtained by using mathematical design equations [4]. Ground plane has length $\mathrm{lg}$, and width $\mathrm{wg}_{\mathrm{g}}$. The tangential components of electric field are in phase. Therefore, maximum radiated field is normal to the surface of the structure. However, normal components of the electric field at the two edges along the width out of phase. Hence, no radiation in broadside direction. As per transmission line model ground plane should be infinite in extent. Practically, ground plane is finite with size greater than the patch dimensions by approximately six times the substrate thickness.

Microstrip feed using quarter wave transformer has been used for feeding the antenna as calculated from equation 1. The input impedance is taken at the base of microstrip feedline and is referred to $50 \Omega$. i.e. Zo.

$$
Z_{q w}=\sqrt{Z_{. r m p a} * Z .0}
$$

where the Wqw and Lqw represent the width and length of the quarter wave transformer as calculated from $\mathrm{Zqw}$ i.e. impedance of quarter wave transformer using txline software by AWR .

The operating frequency range of the designed antenna is $38 \mathrm{THz}$ to $48 \mathrm{THz}$ with centre frequency 43THz. The substrate is RT Duroid $5870\left(\varepsilon_{\mathbf{r}}=2.33\right)$ as shown in Figure 1.

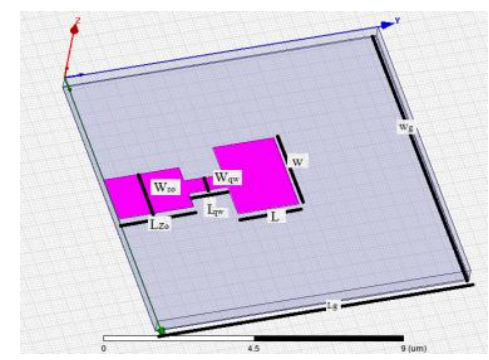

Fig. 1. Rectangular microstrip patch antenna

Using design parameters, the patch antenna is designed in Ansoft HFSS with air box enclosing it with almost 10 times the size of antenna and having radiation boundary as shown in Figure 2.

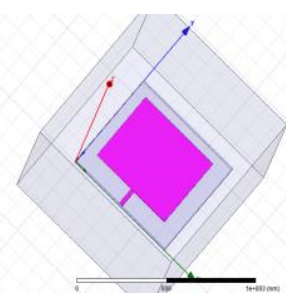

Fig. 2. RMPA design in HFSS

Constructional details are shown in Table 1. 
Table 1. Design parameters of RMPA

\begin{tabular}{c|c}
\hline Frequency range & $\mathbf{3 8 - 4 8 T H z}$ \\
\hline Center frequency & $43 \mathrm{THz}$ \\
$\varepsilon_{\mathrm{r}}$ & 2.33 \\
$\varepsilon_{\mathrm{eff}}$ & 2.04 \\
$\mathrm{~h}$ & $0.5 \mu \mathrm{m}$ \\
$\mathrm{w}$ & $2.7 \mu \mathrm{m}$ \\
$\mathrm{L}_{\mathrm{eff}}$ & $2.44 \mu \mathrm{m}$ \\
$\Delta \mathrm{L}$ & $0.25 \mu \mathrm{m}$ \\
$\mathrm{L}$ & $1.94 \mu \mathrm{m}$ \\
$\mathrm{Lg}_{\mathrm{g}}$ & $4.34 \mu \mathrm{m}$ \\
$\mathrm{W}_{\mathrm{g}}$ & $5.7 \mu \mathrm{m}$ \\
Lqw & $1.25 \mu \mathrm{m}$ \\
Wqw & $0.5 \mu \mathrm{m}$ \\
Lzo & $2.4 \mu \mathrm{m}$ \\
Wzo & $1.6 \mu \mathrm{m}$ \\
\hline
\end{tabular}

\subsection{Simulation results using Ansoft HFSS}

RMPA for ' $\mathrm{S}$ ' shaped MTM has been simulated in HFSS. Figure 3 shows the simulation results where S11 is return loss or reflection coefficient.

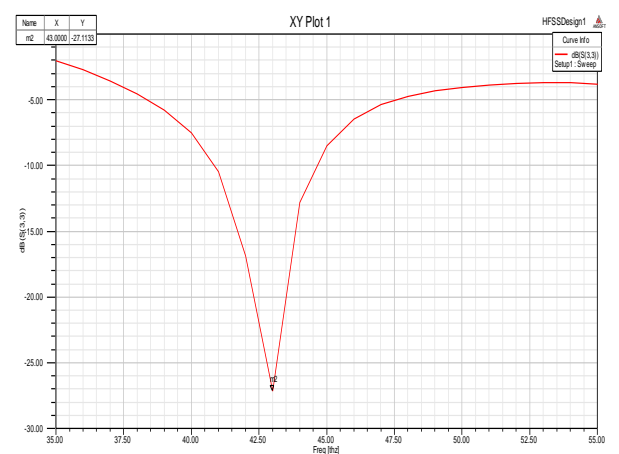

(a)

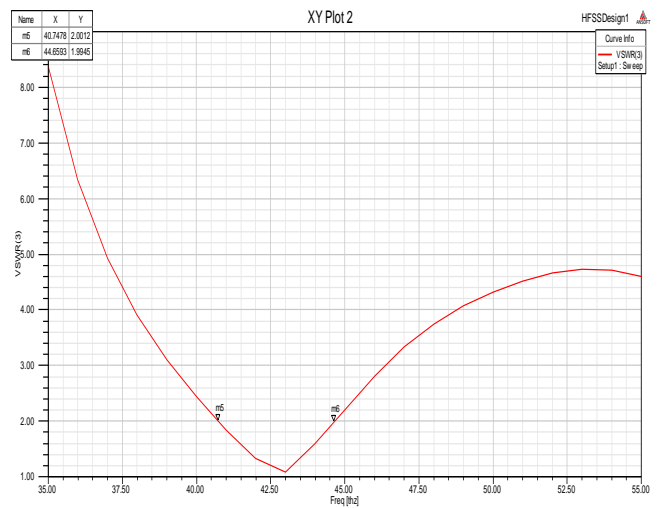

(b)

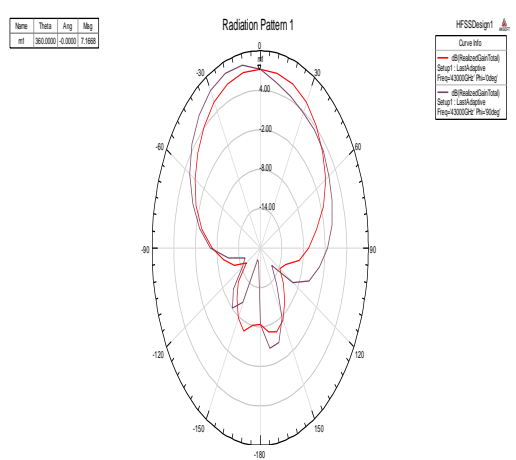

(c)
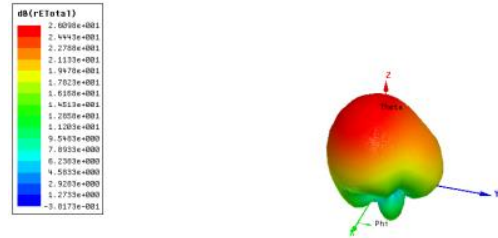

(d)

Fig.3. RMPA for 'S' shaped MTM: a) Return loss b)VSWR c) radiation pattern in $\mathrm{E}$ and $\mathrm{H}$ plane of RMPA d) $3 \mathrm{D}$ radiation plot

Simulation results have been shown in Table 2.

Table 2. Simulation results of RMPA at $42 \mathrm{THz}$

\begin{tabular}{c|c}
\hline Parameters & RMPA \\
\hline $\mathrm{S}_{11}(\mathrm{~dB})$ & -27.11 \\
VSWR & 1.09 \\
Bandwidth $(\mathrm{THz})$ & 3.92 \\
Realized Total Gain in dB & 7.16 \\
Peak directivity $(\mathrm{dB})$ & 6.52 \\
Front to back lobe ratio & 14.38 \\
\hline
\end{tabular}

It can be seen that $\mathrm{S} 11$ is crossing $10 \mathrm{~dB}$ line and VSWR is less than 2. Bandwidth is $3.92 \mathrm{THz}$, which is range of frequencies with VSWR $<2$.

\subsection{Simulation results using Matlab}

Microstrip antennas (often called patch antennas) are widely used in the microwave frequency region because of their simplicity and compatibility with printed-circuit technology, making them easy to manufacture either as stand-alone elements or as elements of arrays [5]. The parametric study using the CAD formulas are fairly accurate for thin substrates and illustrate the basic principles. For thin substrates the CAD formulas may even be accurate enough for final design purposes. For 
thicker substrates these formulas can still be used for initial design work, with full-wave simulation tools used to complete the final design. Using CAD formulas and programming in Matlab by parametric study, results have been shown in Figure 4.

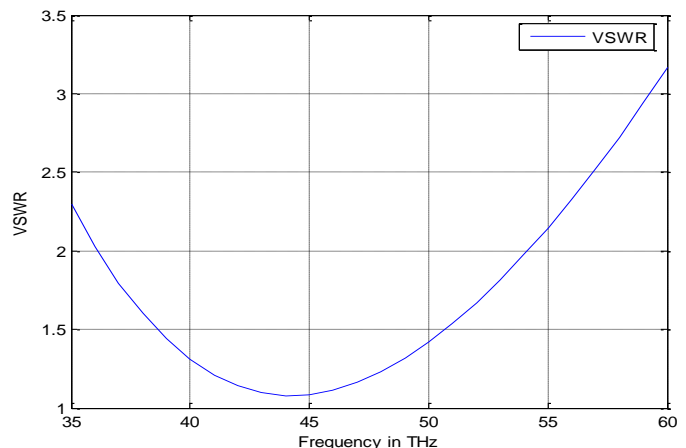

(a)

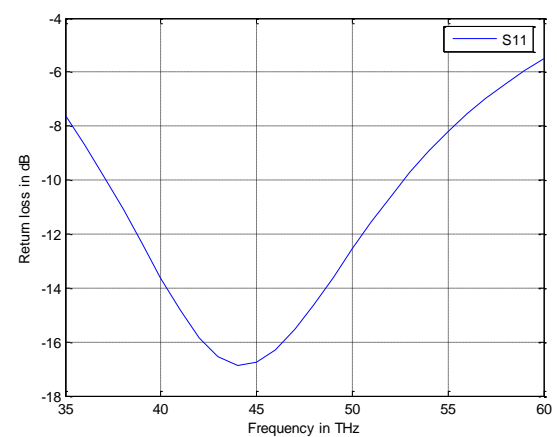

(b)

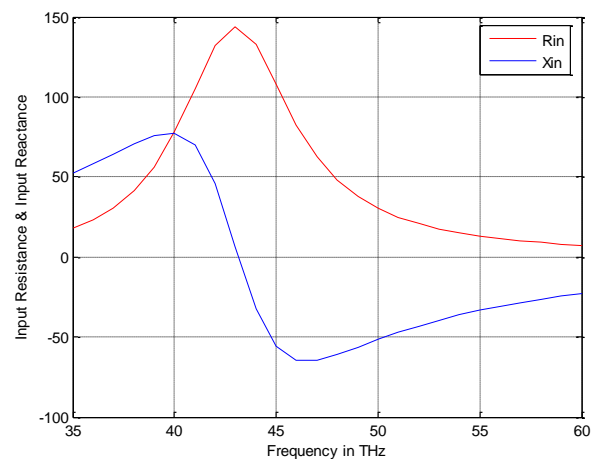

(c)

Fig. 4. RMPA for 'S' shaped MTM: a) VSWR b) Return loss c) Input impedance vs frequency

It has been seen from Figure 4 that using CAD formulas for microstrip patch antenna in Matlab, we can initially plot the curve between input impedance and frequency. Thereby, we can obtain S11 and VSWR by applying the formulas, Reflection coefficient is ratio of (Zin$\mathrm{Zo}) /(\mathrm{Zin}+\mathrm{Zo})$ and VSWR is ratio of $(1+\mathrm{abs}$ (reflection coefficient) $) /(1$-abs(reflection coefficient)).

From the curve of VSWR, the percentage bandwidth can be calculated by the percentage difference of lower and upper frequency bounds with the center frequency. Peak directivity can also be found using CAD formulas. The results obtained by coding in Matlab have been shown in Table 3.

Table 3. Simulation results of RMPA using Matlab

\begin{tabular}{c|cc}
\hline Parameters & Using Matlab & Using HFSS \\
\hline $\mathrm{S}_{11}(\mathrm{~dB})$ & -16.54 & -27.11 \\
VSWR & 1.096 & 1.09 \\
\%Bandwidth & 11.09 & 9.09 \\
Peak directivity $(\mathrm{dB})$ & 8.08 & 6.52 \\
\hline
\end{tabular}

Thus, the results obtained from two different methods of analysis i.e. CAD formulas in Matlab and Finite Element Method in HFSS are well in coherence with each other. This shows that the parametric study using equivalent circuit analysis from which CAD formulas have been derived give nearly parallel results with Finite Element Method.

\section{Proposed S-shaped metamaterial}

In 1968, Russian scientist Veselago [6] postulated a negative material and theoretically proved the phenomenon that a uniform plane-wave followed the left-hand rule in a medium with negative permittivity $(\varepsilon)$ and negative permeability $(\mu)$. The first work in this direction was by Pendry. He created a medium consisting of thin wires arranged in a periodic array [7]. These wires acted as a plasma medium, whereby $\varepsilon$ varies with frequency, Pendry next achieved a negative $\mu$ with a periodic array of metallic loops called Split Ring Resonators [8]. In a medium composed of these rings the permeability, $\mu$, varied with frequency, and could become negative. In 1999, Smith combined the rod and ring materials to finally produce a material with simultaneously negative $\varepsilon$ and $\mu$, a left-handed material [9]. The wire strips affect the $\varepsilon$ and the split-ring resonators (SRRs) alter the $\mu$ of the medium thus giving a frequency dependent negative material with both the parameters negative. The wire medium and the SRRs have certain frequency dependence. The rod gives negative permittivity, $\varepsilon$, and ring material creates a negative permeability, $\mu$, this combined rod and ring material gives negative index of refraction.

$\mathrm{S}$ shaped metamaterial has been proposed having bianisotropy. It behaves as DNG i.e. Double Negative Group.Such materials have negative permittivity and negative permeability in the same frequency region. Thus having negative refraction in the same [10], [11]. 
The parameter retrieval i.e. parameter extraction using $\mathrm{S}$ parameters [12] has been followed using NRW approach to observe the negative refraction region of MTM. The constructional details along with the curve showing negative refraction are as under.

\subsection{Constructional Details}

It is constructionally very simple, consists of a $S$ shaped conductor on top and bottom surface of a RT Duroid Substrate, such that it looks like Figure of eight from top view[13],[14].Thus two opposite S-shaped metallic strips divide the unit cell into three regions[15].Unit cell formed in HFSS is shown in Figure 5(a) with constructional details in Figure 5 (b) where $\sigma \mathrm{s}$ is the resistance of the metallic strips in each loop and $\mathrm{F}$ is to be the fractional volume of the cell occupied by either the top or bottom loop of the ' 8 ' pattern. The dimensions of a unit cell are in the ' $a$ ' in the $x$ direction, ' $b$ ' in the $y$ direction, and ' $l$ ' in the $z$ direction. Thus, in the xy plane, $S=a b$, is the area of a periodic unit. From the structure, we assume F1 to be the fractional volume of the cell occupied by the top loop of the 8 pattern (Area I, Loop 1), F2 to be the fractional volume of the cell occupied by the bottom loop of the 8 pattern (Area II, Loop 2), and F3 to be the fractional volume of the cell not enclosed by the rings (Area III). Therefore, F1 + $\mathrm{F} 2+\mathrm{F} 3=1$.

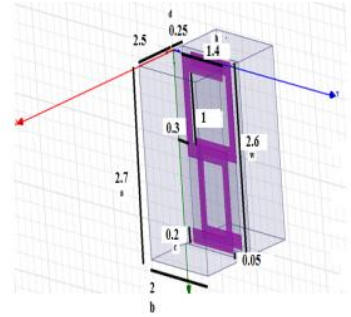

(a)

\begin{tabular}{c|c}
\hline Parameter & $\begin{array}{c}\text { Value } \\
\text { (in } \mu \mathrm{m})\end{array}$ \\
\hline h & 1.4 \\
w & 2.6 \\
a & 2.7 \\
b & 2 \\
c & 0.2 \\
d & 0.25 \\
l & 0.5 \\
\hline
\end{tabular}

(b)

Fig, 5. S shaped MTM a) unit cell designed in HFSS b) constructional details with $\sigma \mathrm{s}=0.5 \Omega$ and $\mathrm{F}=0.3$

\subsection{Simulation Results}

Ansoft HFSS has been used to simulate the unit cell designed in Figure 6(a). Upon simulation it was found that at center frequency $36 \mathrm{THz} \quad \mathrm{S}_{11}$ (reflection coefficient) is $-37.5504 \mathrm{~dB}, \quad \mathrm{~S}_{21}$ (transmission coefficient) is $-1.6905 \mathrm{~dB}$. The boundaries and lumped ports (1 and 2) have been assigned as per Figure 6(a),(b),(c).

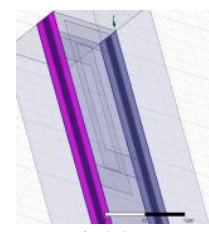

(a)H Field

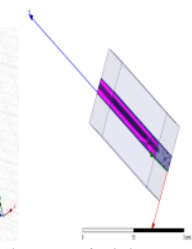

(b) E Field

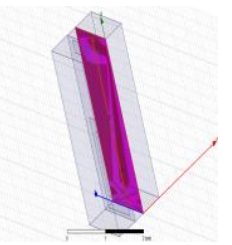

(c) Lumped Port
Fig. 6.a) $\mathrm{H}$ field b) E field specifying boundary condition c) Lumped Port

Nicolson Ross Wier method has been used to calculate the material properties from transmission and reflection coefficients.

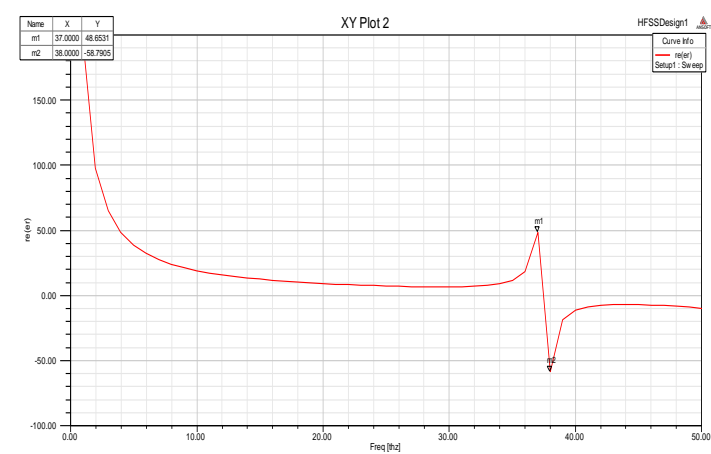

(a)

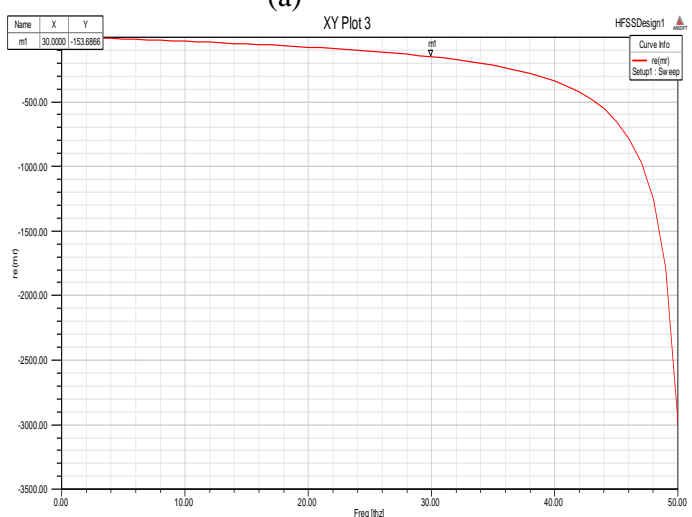

(b) 


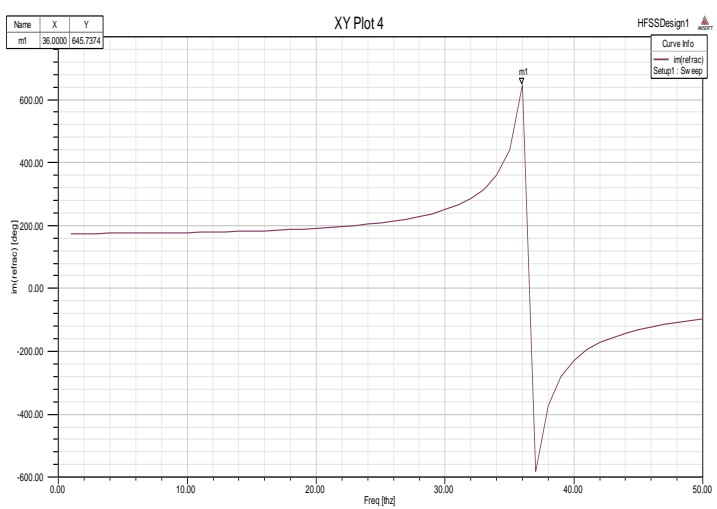

(c)

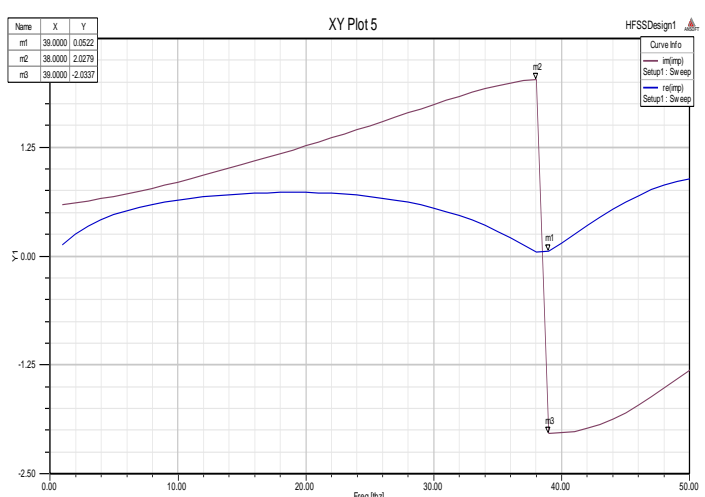

(d)

Fig. 7. a) Resonance in permeability from $37.5 \mathrm{THz}$ to $54 \mathrm{THz}$ b) Plasma frequency of permittivity is $37.5 \mathrm{THz}$ c) Negative refraction from $37.5 \mathrm{THz}$ to $54 \mathrm{THz}$ d) Real part of impedance is positive from $37.5 \mathrm{THz}$ to $54 \mathrm{THz}$

It can be observed as in Figure 7 (a), (b), (c), (d) that region of negative permeability extends from $37.5 \mathrm{THz}$ to around $54 \mathrm{THz}$, the region of negative permittivity extends from $37.5 \mathrm{THz}$ onwards, region of negative refraction can also be observed from $37.5 \mathrm{THz}$ to around $54 \mathrm{THz}$, in the same region positive real impedance can also be noted.

\subsection{Mathematical Proof}

S shaped MTM has magnetic properties because of internal inductances and capacitances. It can be simplified in terms of two magnetic loops as shown in Figure 8(a). Using transmission line theory we can draw its equivalent circuit as in Figure 8 (b).
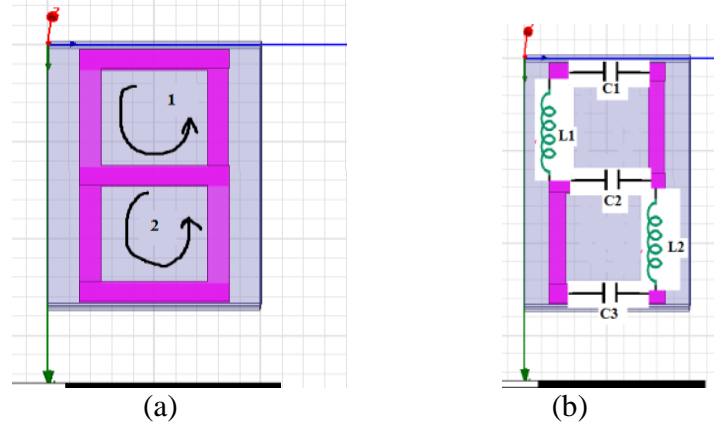

(b)

Fig. 8. S-shaped MTM a) magnetic circuit loops b) equivalent circuit

As seen from Figure 8 (b), $\mathrm{C}_{2}$ is the capacitance between the center metallic strips, and $\mathrm{C}_{1}$ and $\mathrm{C}_{3}$ are the capacitances of the top and bottom metallic strips respectively. Take the inductance per unit length in the $\mathrm{z}$ direction of each half ring to be $\mathrm{L}_{1}$ and $\mathrm{L}_{2}$. Assume $\mathrm{C}_{1}=\mathrm{C}_{3}=\mathrm{C}$ and $\mathrm{L}_{1}=\mathrm{L}_{2}$ and $\mathrm{F} 1=\mathrm{F} 2=\mathrm{F} 3=0.3=\mathrm{F}$.

Let us solve the circuit by finding equivalent inductance and capacitances i.e. $\mathrm{L}_{\mathrm{eq}}$ and $\mathrm{C}_{\mathrm{eq}}$.

$\mathrm{L}_{\mathrm{eq}}$ is given by the area enclosed by each ring i.e.

$$
L_{e q}=\frac{\mu_{0} \cdot F \cdot S}{l}
$$

From equation $2, \mathrm{~L}=4.07 * 10^{-6} \mathrm{H}$

Using simple circuit theory, equivalent capacitance $\mathrm{C}_{\mathrm{eq}}$ can be found out as

$$
C_{e q}=\frac{1}{\frac{1}{C}+\frac{1}{\frac{C_{2}}{2}}}
$$

Thus, resonant frequency of S shaped MTM is given by using 2 and 3 as

$$
\omega_{m o}=\sqrt{\frac{1}{L_{e q} \cdot C_{e q}}}
$$

Therefore, using equation 2 and 3 in 4 we get

$$
\omega_{\text {то }}=\sqrt{\frac{1}{L \frac{1}{\frac{1}{C}+\frac{1}{C_{2} / 2}}}}
$$

Now the capacitance between two metallic strips can be 
calculated as

$$
C=C_{2}=\varepsilon_{0} \frac{h c}{d}+\varepsilon_{0} \frac{h c}{l-d}
$$

where $\varepsilon_{0}$ is the permittivity of free space.

Putting values from Figure 5 (b)in equation 6 we get, $\mathrm{C}=1.983 * 10^{-23} \mathrm{~F}$.

Substituting values of $\mathrm{C}$ and $\mathrm{L}$ in equation 4 , we get magnetic resonance frequency, $\mathrm{f}_{0}=31 \mathrm{THz}$.

Also, the magnetic plasma frequency is given by

$$
\omega_{m p}=\omega_{m o} \sqrt{\frac{1}{1-2 F}}
$$

Upon substitution, $\mathrm{fp}=49 \mathrm{THz}$.

Experimentally the value of magnetic resonance frequency has been found out to be $37.5 \mathrm{THz}$ and theoretically the value is $31 \mathrm{THz}$. So, there is around $17 \%$ error in the simulation.

This rectangular microstrip patch antenna has been fabricated by scaling (by $(1 / 10000)$ the above designs to low frequency. The substrate has also been changed to FR4. The fabricated antenna is shown in Figure.9.

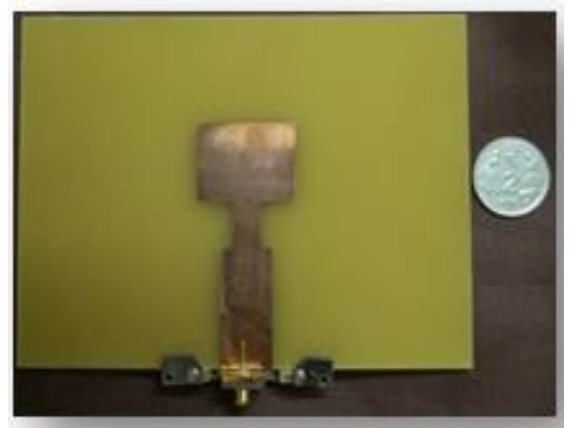

Fig. 9. Fabricated Antennas: Microstrip Patch antenna

The above design is simulated in HFSS and results are shown in Figures 10.

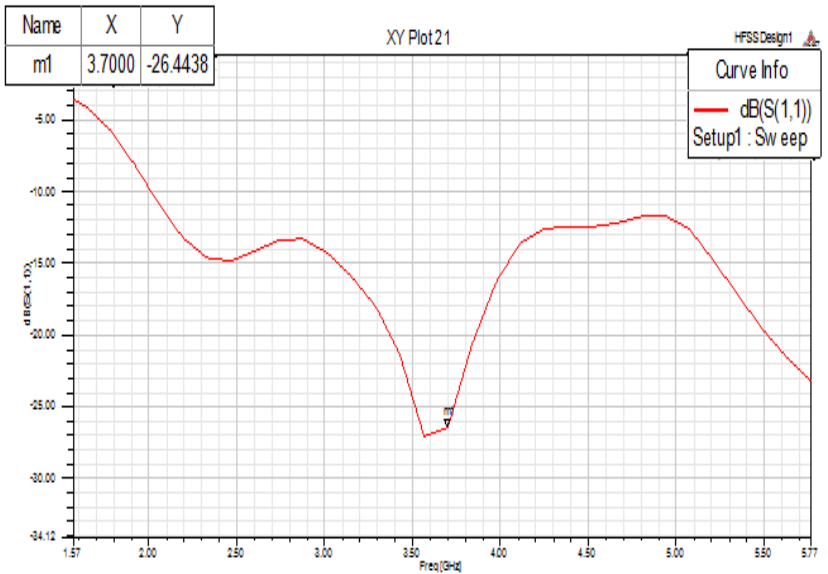

(a)

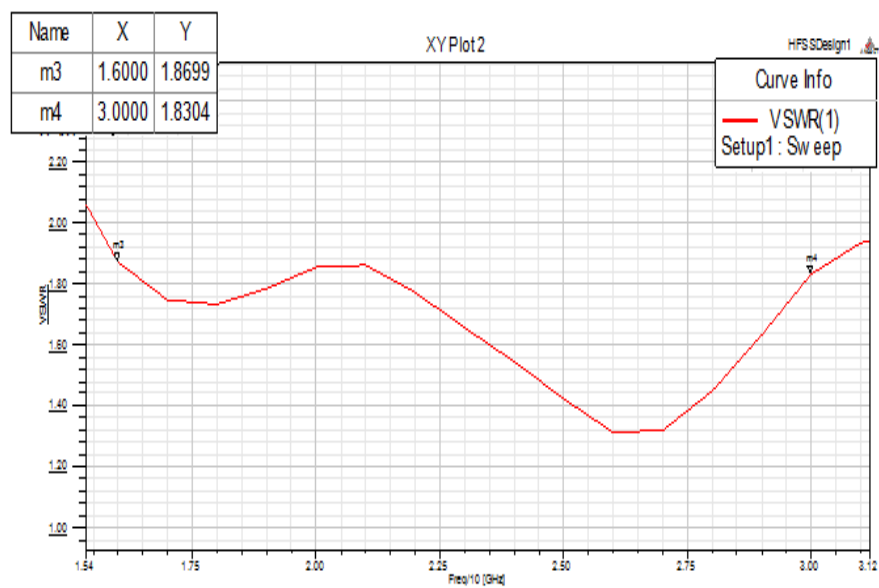

(b)

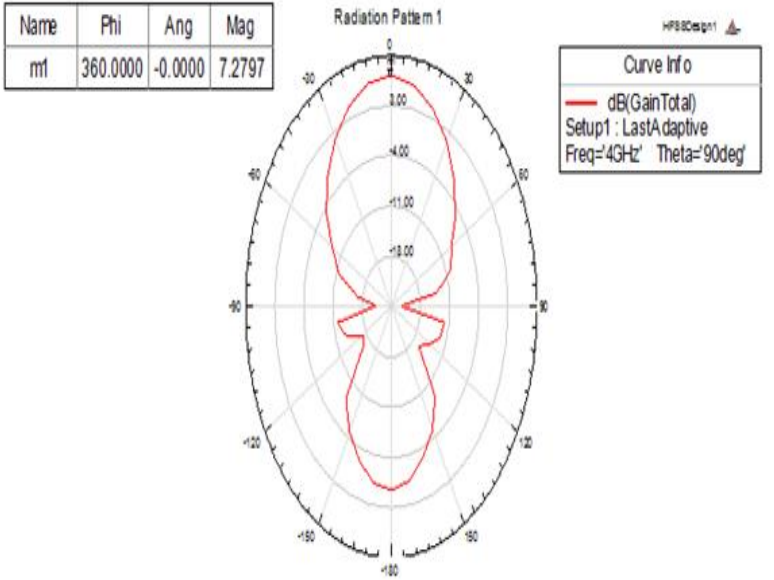

(c)

Fig.10. Patch Antenna: a) Return Loss b ) VSWR c) Radiation Pattern

Using Keysight RF Fieldfox Network Analyzer as shown in Figure 11, the simulation and fabrication results are compared in Figure.12. 


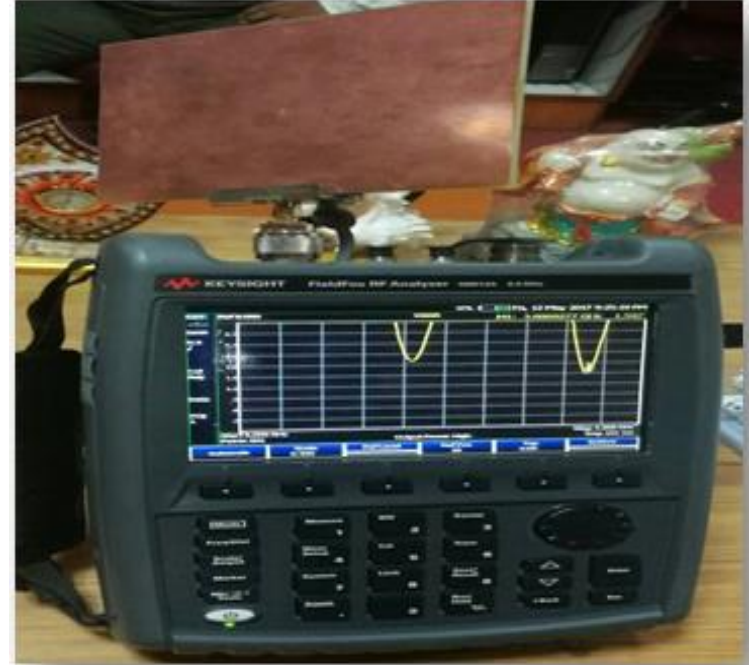

Fig..11. Keysight Fieldfox RF Network Analyzer

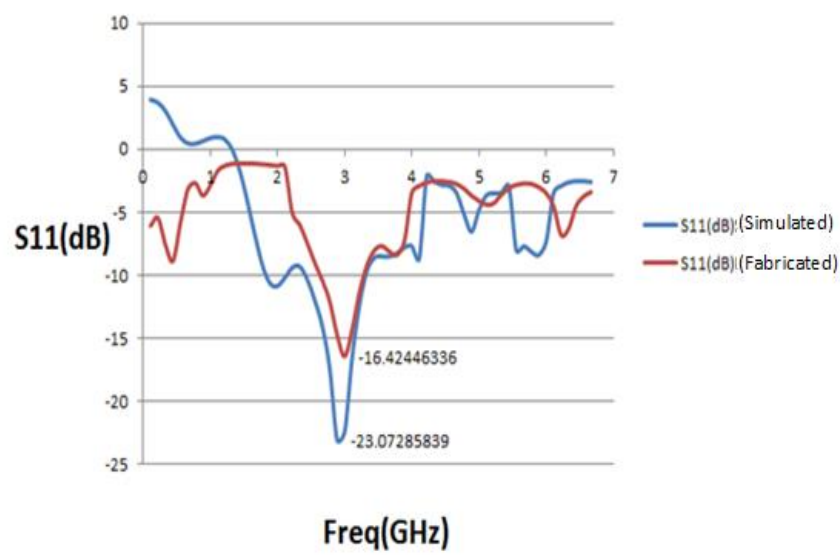

Fig. 12. Return Loss of Patch Antenna

Using gain measurement set-up as shown in Figure 13, gain and radiation pattern have been obtained in Figure 14.

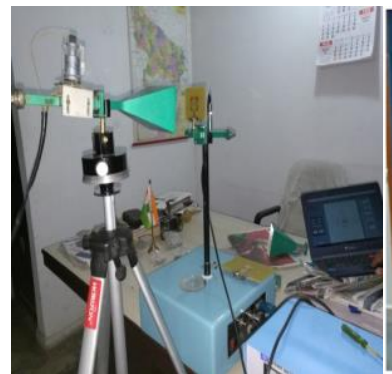

(a)

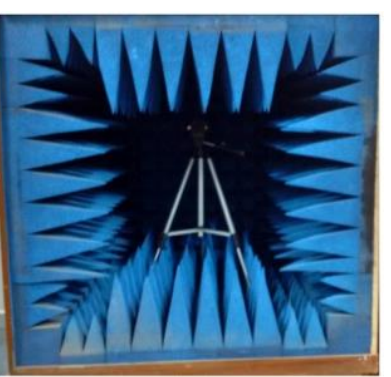

(b)
Fig. 13. (a) Gain measurement set up for $\mathrm{X}$ band (b) Set up inside anechoic chamber
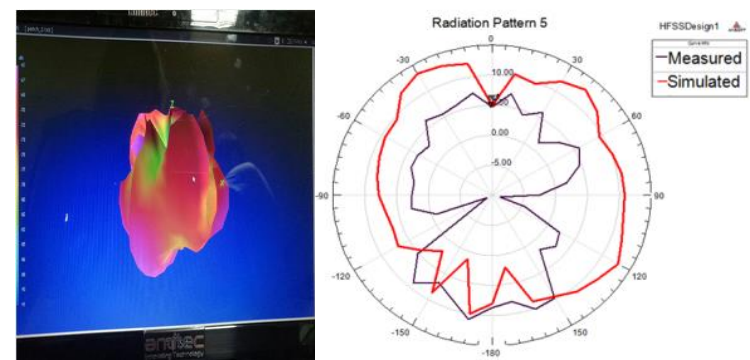

Fig. 14. Radiation Pattern measurement of Patch Antenna

The above results have been tabulated in Table 4 .

Table 4. Comparison of simulated and fabricated results for RMPA

\begin{tabular}{c|cccc}
\hline $\begin{array}{c}\text { Antenna } \\
\text { Design }\end{array}$ & $\begin{array}{c}\text { Gain } \\
(\mathbf{d B})\end{array}$ & $\begin{array}{c}\text { Direc- } \\
\text { tivity } \\
(\mathbf{d B})\end{array}$ & $\begin{array}{c}\text { Band- } \\
\text { width } \\
(\mathbf{G H z})\end{array}$ & $\begin{array}{c}\text { Return } \\
\text { loss } \\
(\mathbf{d B})\end{array}$ \\
\hline $\begin{array}{c}\text { Patch } \\
\text { Antenna }\end{array}$ & 1.9 & 5 & 1.3 & 23 \\
$\begin{array}{c}\text { Simulated }) \\
\text { Patch } \\
\text { antenna }\end{array}$ & 1.7 & 4 & 1.5 & 16 \\
(Fabricated) & & & & \\
\hline
\end{tabular}

Thus, "fabricated and simulated antennas" helps in achieving miniaturization and bandwidth enhancement which is in coherence with the proposed antenna configurations with nearly $10 \%$ error.

\section{Antenna Parameter Optimization}

\subsection{Design}

Antenna is characterized by different parameters e.g. gain, bandwidth, VSWR3dB beam width in E,H plane, return loss. These parameters have been obtained for RMPA as in table 2 using HFSS. Parameter optimization is done by embedding the proposed $S$ shaped MTM 3X4 array as shown in Figure 15, having $0.55 \mu \mathrm{m}$ as $\Delta \mathrm{x}$ and $1.3 \mu \mathrm{m}$ as $\Delta \mathrm{y}$ inside the RMPA. 


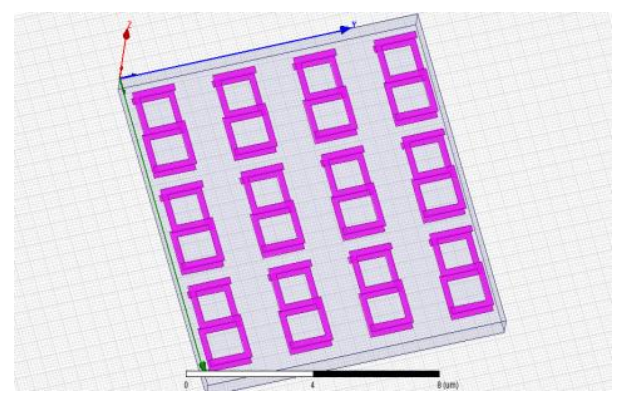

Fig. 15. Embedding S-shaped MTM array inside RMPA substrate

Inserting proposed MTMs 3X4 array inside the RMPA substrate, such that the top patch of MTM is $0.125 \mu \mathrm{m}$ down from the top of the substrate, will change the effective material parameters below the patch, thereby affecting the performance of the antenna.

\subsection{Simulation}

Simulation results for MTM Antenna with ' $S$ ' shaped MTM Array are shown in Figure 16.

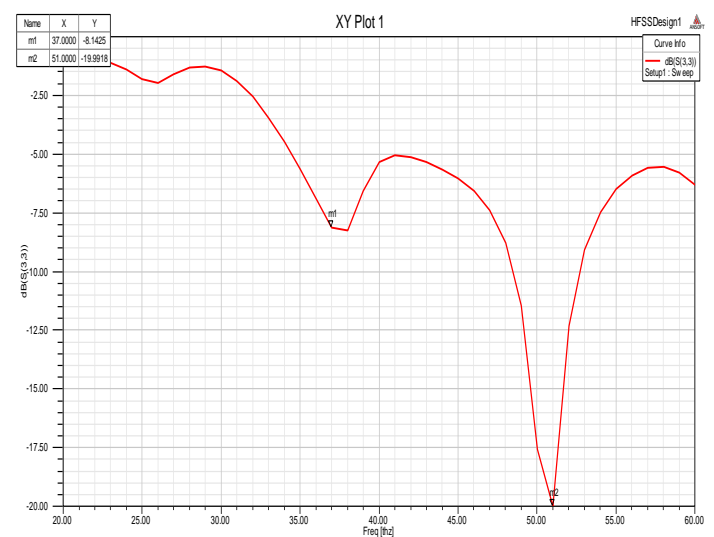

(a)

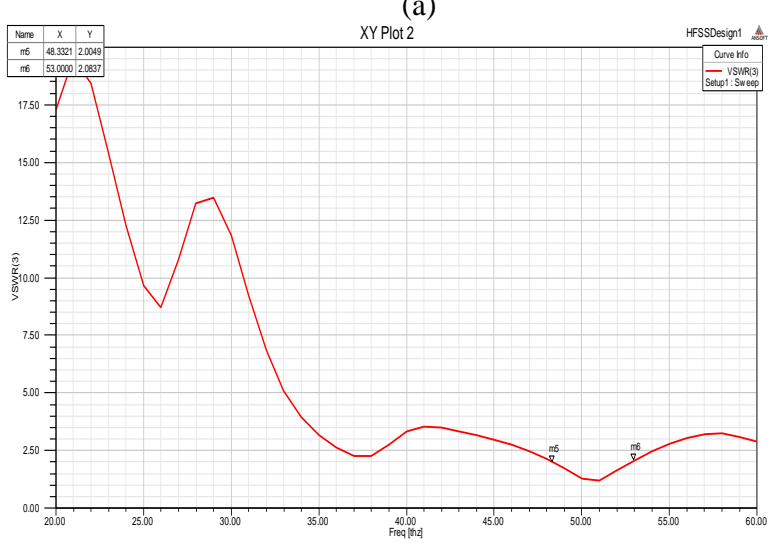

(b)
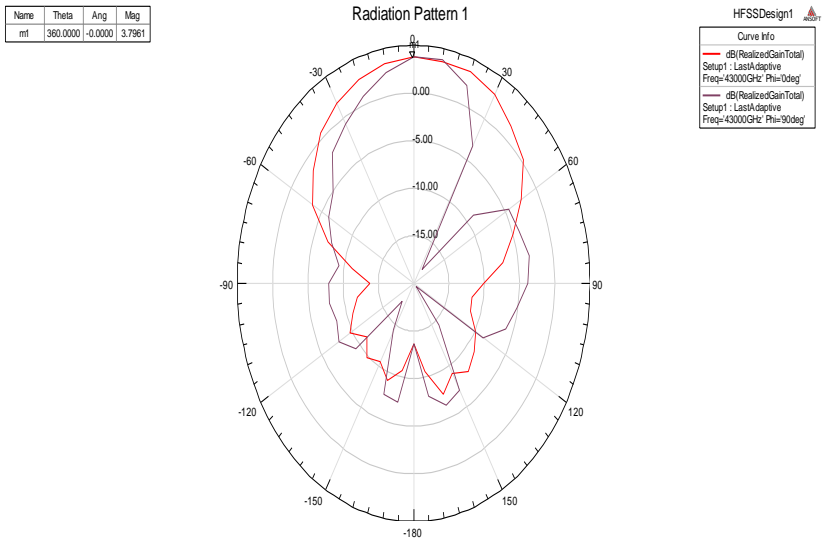

(c)
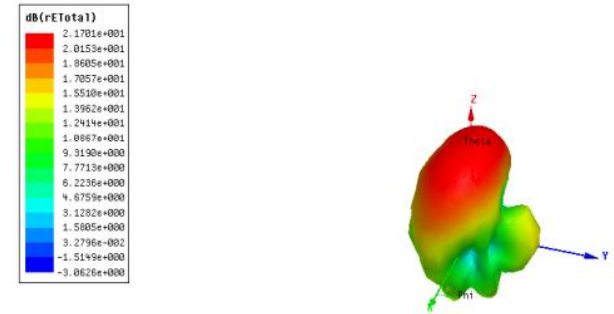

(d)

Fig. 16. MTM Antenna: a) Return loss b) VSWR c) Radiation pattern in $\mathrm{E}$ and $\mathrm{H}$ plane of RMPA d) 3D radiation plot

Table 5 gives the obtained simulated values of parameters and comparison of S-shaped MTM RMPA with RMPA without MTM.

Table 5: Simulation and comparison results of S-shaped MTM embedded in RMPA substrate

\begin{tabular}{c|cc}
\hline $\begin{array}{c}\text { ANTENNA/ } \\
\text { Parameters }\end{array}$ & RMPA & $\begin{array}{c}\text { RMPA with S- } \\
\text { shaped MTM }\end{array}$ \\
\hline S $_{11}(\mathrm{~dB})$ & -27.11 & -19.99 \\
VSWR & 1.09 & 1.22 \\
Bandwidth (in THz) & 3.92 & 4.67 \\
Realized Total Gain in & 7.16 & 3.79 \\
dB & & \\
Peak Directivity(dB) & 6.52 & 7.23 \\
Front to back lobe & 14.38 & 19.91 \\
ratio(dB) & & \\
\hline
\end{tabular}

Upon designing and analyzing proposed ' $\mathrm{S}$ ' shaped MTM array inside RMPA substrate the results are tabulated as in Table 5. It can be seen that there is enhancement in bandwidth with S-shaped MTM by 
$770 \mathrm{GHz}$. VSWR decreases up to $11 \%$. The smaller the VSWR is, the better the antenna is matched to the transmission line and the more power is delivered to the antenna. Decrease in realized total gain as observed from the radiation pattern is $89 \%$. Directivity improves by $43 \%$ and thereby reducing side lobes as can be observed from front-to-back lobe ratio of the antenna. The S-shaped metamaterial antenna has been fabricated by scaling (by $(1 / 10000)$ the above designs to low frequency. The substrate has also been changed to FR4. The fabricated antenna is shown in Figure 17.

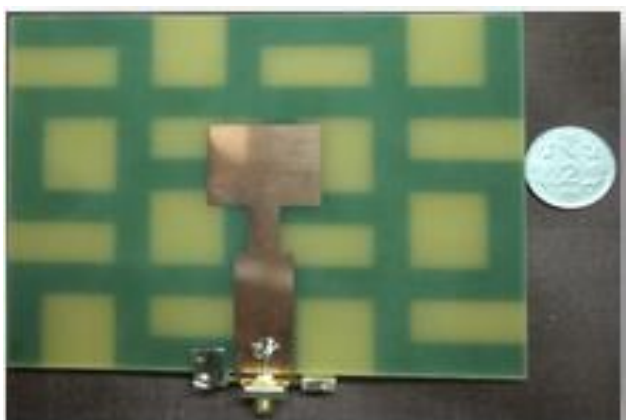

Fig. 17. Fabricated Antennas of Swastika metamaterial Antenna

The above structure is designed and simulated in HFSS. The simulation results are shown in Figures 18.

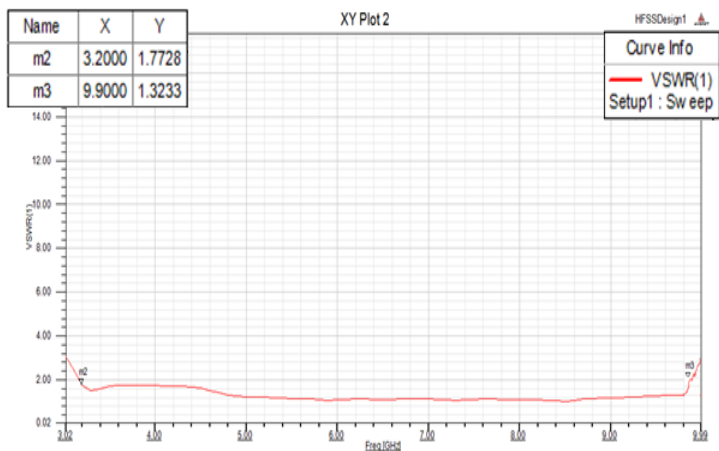

(a)

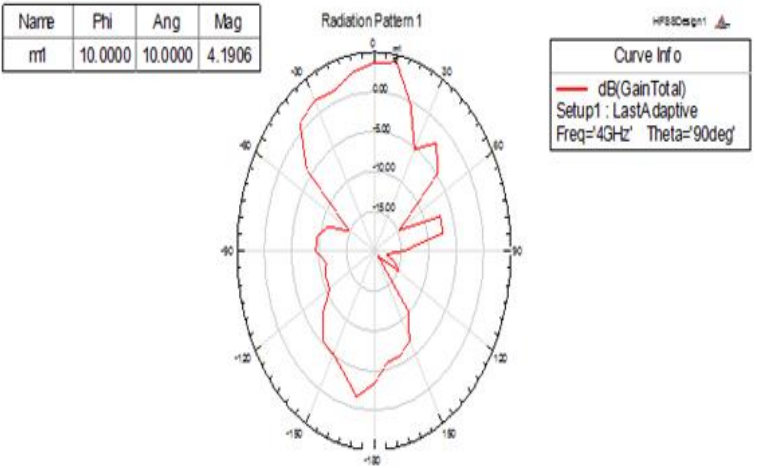

(b)

Figure 18: S-shaped Metamaterial Patch Antenna: a) VSWR b) Radiation Pattern
The return loss and VSWR curves are studied using Keysight RF Fieldfox Network Analyzer as shown in Figure 19.

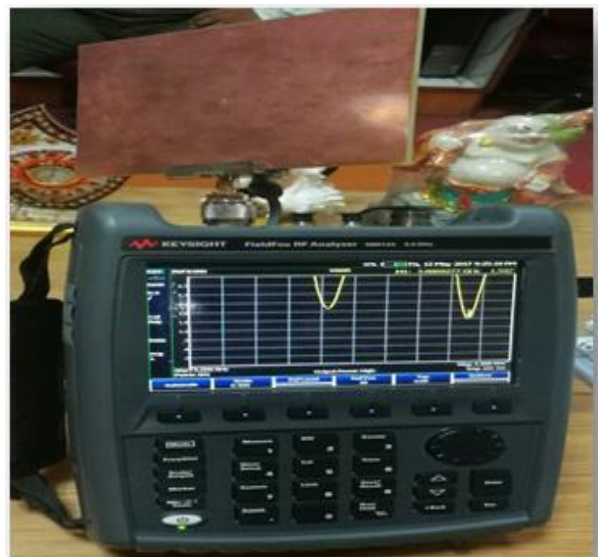

Fig. 19. Keysight Fieldfox RF Network Analyzer

The simulation and fabrication results of return loss are compared in Figure 20.

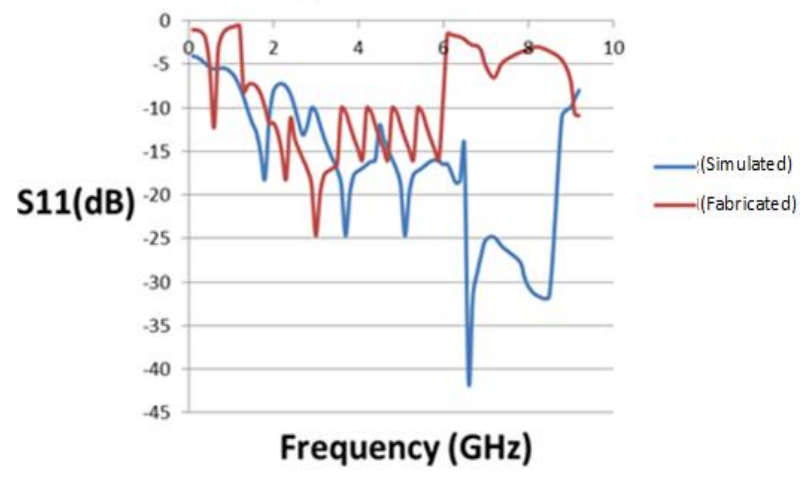

Fig. 20. Return Loss of S-shaped metamaterial Patch Antenna

Using gain measurement set-up radiation pattern has been obtained in Figure 21

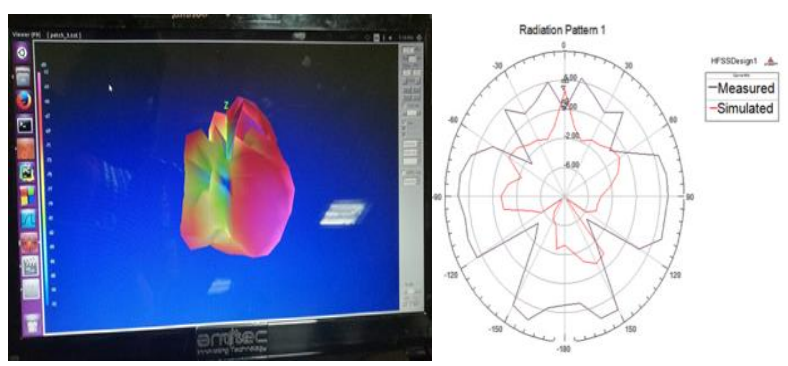

Fig. 21. Radiation Pattern measurement of Swastika metamaterial patch Antenna

The above results have been tabulated in Table 6 . 
Table 6. Comparison of simulated and fabricated results for Sshaped metamaterial RMPA

\begin{tabular}{|c|c|c|c|c|c|}
\hline $\begin{array}{c}\text { Antenna } \\
\text { Design }\end{array}$ & $\begin{array}{l}\text { Gain } \\
\text { (dB) }\end{array}$ & $\begin{array}{l}\text { Direc } \\
\text {-tivity } \\
\text { (dB) }\end{array}$ & $\begin{array}{c}\text { Band- } \\
\text { width } \\
\text { (GHz } \\
\text { ) }\end{array}$ & $\begin{array}{c}\text { Return } \\
\text { loss } \\
\text { (dB) }\end{array}$ & $\begin{array}{c}\text { Minia } \\
\text {-turi- } \\
\text { zation } \\
(\%)\end{array}$ \\
\hline $\begin{array}{c}\text { S-shaped } \\
\text { meta- } \\
\text { material } \\
\text { RMPA, } \\
\text { Simulated }\end{array}$ & 4.9 & 5 & 6.7 & 20 & 51 \\
\hline $\begin{array}{c}\text { S-shaped } \\
\text { meta- } \\
\text { material } \\
\text { RMPA, } \\
\text { Fabricated }\end{array}$ & 4.8 & 4 & 4.2 & 25 & 84 \\
\hline
\end{tabular}

Thus, "fabricated and simulated antennas" helps in achieving miniaturization and bandwidth enhancement which is in coherence with the proposed antenna configurations with $12 \%$ error.

\section{Trade-offs}

In this paper, around $17 \%$ error has been computed between numerical simulations and theoretical calculations of $\mathrm{S}$-shaped metamaterial. Using MTM array (3X4) inside RMPA, it can be seen that there is enhancement in bandwidth by $770 \mathrm{GHz}, 11 \%$ reduction in VSWR and directivity increases by $11 \%$. However, reduction in gain has also been observed. Also, no improvement in return loss can be seen. The increase in directivity accounts for reduction in front to back lobe ratio.

However, the coherence between equivalent circuit analysis using parametric study by applying CAD formulas in Matlab and Finite Element Method technique in HFSS has been observed.

Thus, we have been able to obtain more bandwidth, better and enhanced directivity at cost of realized total gain and VSWR for patch antenna with proposed MTM $3 \mathrm{X} 4$ array.

\section{References}

1. H. Schantz, The Art and Science of Ultra Wide Band Antennas, Artech House, 2005.

2. C.A.Balanis, Antenna Theory, John Wiley \& Sons, Inc., 1997.

3. D.M.Pozar, Microstrip antenna, Proc. IEEE, Vol. 80, 79-81, 1992.

4. Volakis,Antenna Engineering Handbook, McGraw Hills, Chapter-7, pp: 147574-5.

5. S.Wang, A.P. Feresidis, G. Goussetis, J.C. Vardaxoglou, Low-profile resonant cavity antenna with artificial magnetic conductor ground plane, Electronics Letters, 2004,40: 405-406.

6. M.R.Ahsan, M.T. Islam, M.H. Ullah, N. Misran, Bandwidth Enhancement of a Dual Band Planar Monopole Antenna Using Meandered Microstrip Feeding, The Scientific World Journal ,2014.

7. K.F. Lee, K.M. Luk, K.M. Mak, S.L.S Yang, On the Use of U-Slots in the Design of Dual-and TripleBand Patch Antennas, IEEE Antennas and Propagation Magazine, 2011, 53: 60-74.

8. K.L. Wong, Compact and Broadband Microstrip Antennas, Hoboken, NJ, USA: Wiley-Interscience, 2004.

9. Parul Dawar, N.S. Raghava, Asok De, Ultra Wide Band, Multi-resonance Antenna using swastika metamaterial, International Journal of Microwave and Optical Technology,Vol 11, No. 6,Nov. 2016.

10. Md Rezwanul Ahsan, Md. Tariqul Islam, Md. Habib Ullah, Mandeep Jit Singh, Mohd Tarmizi Ali, Metasurface Reflector (MSR) Loading for High Performance Small Microstrip Antenna Design, PLOS One, May 2015. 